International Journal of Pure and Applied Mathematics

Volume 103 No. 3 2015, 439-451

ISSN: 1311-8080 (printed version); ISSN: 1314-3395 (on-line version)

url: http://www.ijpam.eu

doi: http://dx.doi.org/10.12732/ijpam.v103i3.6

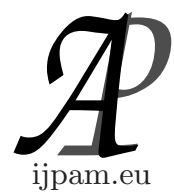

\title{
A CLASS OF KOLMOGOROV SYSTEM WITH EXACT ALGEBRAIC LIMIT CYCLES
}

S. Benyoucef ${ }^{1} \S$, A. Bendjeddou ${ }^{2}$

${ }^{1}$ Department of Mathematics

Faculty of Sciences

University of 20 août

1955, Skikda, ALGERIA

${ }^{2}$ Department of Mathematics

University of Setif 1

19 000, ALGERIA

Abstract: A class of Kolmogorov differential system is introduced, we show that under suitable assumptions, one, two or four algebraic limit cycles can occur, these limit cycles are analytically given.

AMS Subject Classification: 34C25, 34C05

Key Words: Kolmogorov differential systems, invariant curves, periodic solutions, algebraic limit cycles

\section{Introduction}

The second part of sixteenth problem of Hilbert still persists a research area. It proposed to find the maximum number of limit cycles of the differential system:

$$
\left\{\begin{array}{l}
\dot{x}=\frac{d x}{d t}=P(x, y), \\
\dot{y}=\frac{d y}{d t}=Q(x, y),
\end{array}\right.
$$

where $P$ and $Q$ are two polynomials.

Received: April 21, 2015

(C) 2015 Academic Publications, Ltd.

$\S$ Correspondence author url: www.acadpubl.eu 
The topic of limit cycles is interesting both in mathematics and in science and many models from physics, engineering, chemistry, biology, economics..., were displayed as differential systems with limit cycles.

The analysis of the existence, number and stability of limit cycles, have been several articles ( see for instance T.R. Blows and N.G. Lloyd [5], F. Dumortier, J. Llibre and J. Artés [10], H. Giacomini, J. Llibre and M. Viano [11], J. Chavarriga, H. Giacomini and J. Giné [7], J. Giné, J. Llibre[12], [13], J. Llibre[18], [19],[20], Y. Ye[25], X. Zhang[27], M.J. Alvarez, A. Gasull, R. Prohens [2]) and references therein.

Many mathematical models in biology science and population dynamics, frequently involve the systems of ordinary differential equations having the form

$$
\left\{\begin{array}{l}
\dot{x}=\frac{d x}{d t}=x F(x, y), \\
\dot{y}=\frac{d y}{d t}=y G(x, y),
\end{array}\right.
$$

$x(t)$ and $y(t)$ represent the population density of two species at time $t$, and $F(x, y), G(x, y)$ are the capita growth rate of each specie, usually, such systems are called Kolmogorov systems.

Kolmogorov models are widely used in ecology to describe the interaction between two populations, and a limit cycle corresponds to an equilibrium state of the system.

In mathematical modeling of ecological systems and population dynamics, more mathematicians and scientists were attracted to the subject and several results have been published, May[23], Kuang and Freedman [17], X. Huang, Y. Wang, A. Cheng [16], L. Perko[24], and others.

When $F(x, y)$ and $G(x, y)$ are polynomials of degrees $\geq 2$, limit cycles can occur and there is an extensive literature dealing with their existence, number and stability (see for instance Lloyd, Pearson,Saèz and Szántó[21], X.C. Huang, L. Zhu [14], X.C. Huang[15], S. Boqian and L. Demeng [6], Cheng [8], and references therein), but to our knowledge, the exact analytic expressions of the limit cycles for a given kolmogorov system is still unknown even in simplest and specific cases.

This paper is a contribution in that direction, determine the number of limit cycles and give their explicit form.

Motivated by the recent publication of some research papers exhibiting planar polynomial systems with one or more algebraic limit cycles analytically given (see for instance M. Abdelkadder[1], A. Bendjeddou and R. Cheurfa[3], [4], S. Chengbin, S. Boqian[9], Lloyd, Pearson,Saèz and Szántó[22], Peng Yue- 
hui[26] and references therein), we will study the existence and the number of limit cycles of a class of Kolmogorov system, and give their explicit form.

\section{Introductory Concepts}

Let us recall some useful notions.

For $U \in \mathbb{R}[x, y]$, the algebraic curve $U=0$ is called an invariant curve of the polynomial system (2), if for some polynomial $K \in \mathbb{R}[x, y]$ called the cofactor of the algebraic curve, we have

$$
x F(x, y) \frac{\partial U}{\partial x}+y G(x, y) \frac{\partial U}{\partial y}=K U .
$$

The curve $\Gamma=\left\{(x, y) \in \mathbb{R}^{2}: U(x, y)=0\right\}$ is nonsingular of system (2) if the equilibrium points of the system that satisfy

$$
\left\{\begin{array}{l}
x F(x, y)=0, \\
y G(x, y)=0,
\end{array}\right.
$$

are not contained on the curve $\Gamma$.

The curve $U=0$ is formed by trajectories of the system (2), and if the curve is nonsingular, the equilibrium points of the system are contained either in its unbounded components or are located on the curve $K=0$.

A limit cycle $\gamma=\{(x(t), y(t)), t \in[0, T]\}$, is a $T$-periodic solution isolated with respect to all other possible periodic solutions of the system.

We construct here a multi-parameter planar differential system admitting the components of curve

$$
\begin{aligned}
& \Gamma=\left\{(x, y) \in \mathbb{R}^{2}: a x^{2 n}+b x^{n}+c y^{2 m}+d y^{m}+h=0,\right. \\
& \left.(n, m) \in \mathbb{N}^{*} \times \mathbb{N}^{*}\right\},
\end{aligned}
$$

as limit cycles if some conditions on the parameter are satisfied.

\section{The Main Result}

As a main result, we have the following theorem

Theorem 1. The system

$$
\begin{aligned}
& \dot{x}=x\left((x y+P(y))\left(a x^{2 n}+b x^{n}+c y^{2 m}+d y^{m}+h\right)-d m y^{m}-2 c m y^{2 m}\right), \\
& \dot{y}=y\left((x y+Q(x))\left(a x^{2 n}+b x^{n}+c y^{2 m}+d y^{m}+h\right)+b n x^{n}+2 a n x^{2 n}\right),
\end{aligned}
$$


where $a, c$ are positive real, $b, d$ are negative real, $h$ satisfied

$$
\max \left\{\frac{b^{2}}{4 a}, \frac{d^{2}}{4 c}\right\}<h<\frac{b^{2}}{4 a}+\frac{d^{2}}{4 c}, \quad(n, m) \in \mathbb{N}^{*} \times \mathbb{N}^{*},
$$

$P(y)$ and $Q(x)$ are polynomial of any degree, or more general these are analytic functions.

This system admits:

One limit cycle when $n$ and $m$ are odd numbers.

Two limit cycles when one of number $n$ and $m$ is odd and the other is even.

Four limit cycles when $n$ and $m$ are even numbers.

The limit cycles are represented by the curve $\Gamma$.

Proof. We will prove that $\Gamma$ is nonsingular composed of oval and it is an invariant curve of system (6), and

$$
\int_{0}^{T} \operatorname{div}(\Gamma) d t \neq 0 .
$$

i) The curve $\Gamma$ is nonsingular of system (6) composed by oval.

We recall that the curve $\Gamma$ is nonsingular of system (6) if the following system has no real solution.

$$
\begin{aligned}
& a x^{2 n}+b x^{n}+c y^{2 m}+d y^{m}+h=0, \\
& -m x y^{m}\left(d+2 c y^{m}\right)=0, \\
& n y x^{n}\left(b+2 a x^{n}\right)=0 .
\end{aligned}
$$

Note that the curve $\Gamma$ does not intersect the axes: $c y^{2 m}+d y^{m}+h \neq 0$ because $h>\frac{d^{2}}{4 c}$ and $a x^{2 n}+b x^{n}+h \neq 0$ because $h>\frac{b^{2}}{4 a}$,

Then the possible critical points on $\Gamma$ are:

$$
\begin{gathered}
A_{1}\left(-\sqrt[n]{\frac{-b}{2 a}},-\sqrt[m]{\frac{-d}{2 c}}\right), A_{2}\left(-\sqrt[n]{\frac{-b}{2 a}}, \sqrt[m]{\frac{-d}{2 c}}\right) \\
A_{3}\left(\sqrt[n]{\frac{-b}{2 a}},-\sqrt[m]{\frac{-d}{2 c}}\right), A_{4}\left(\sqrt[n]{\frac{-b}{2 a}}, \sqrt[m]{\frac{-d}{2 c}}\right),
\end{gathered}
$$

when $m$ and $n$ are even numbers.

$$
A_{4}\left(\sqrt[n]{\frac{-b}{2 a}}, \sqrt[m]{\frac{-d}{2 c}}\right)
$$


when $m$ and $n$ are odd numbers.

$$
A_{2}\left(-\sqrt[n]{\frac{-b}{2 a}}, \sqrt[m]{\frac{-d}{2 c}}\right), A_{4}\left(\sqrt[n]{\frac{-b}{2 a}}, \sqrt[m]{\frac{-d}{2 c}}\right)
$$

when $m$ is odd and $n$ is even.

$$
A_{3}\left(\sqrt[n]{\frac{-b}{2 a}},-\sqrt[m]{\frac{-d}{2 c}}\right), A_{4}\left(\sqrt[n]{\frac{-b}{2 a}}, \sqrt[m]{\frac{-d}{2 c}}\right)
$$

when $m$ is even and $n$ is odd.

All the points do not lie on $\Gamma$, as $h \neq \frac{b^{2}}{4 a}+\frac{d^{2}}{4 c}$, and hence the curve is nonsingular.

Now we prove that $\Gamma$ is composed by ovals.

We consider the equation (5) as

$$
c z^{2}+d z+\left(a x^{2 n}+b x^{n}+h\right)=0, \text { where } z=y^{m} .
$$

The discriminant

$$
\Delta=d^{2}-4 c\left(a x^{2 n}+b x^{n}+h\right)=-4 a c v^{2}-4 b c v+d^{2}-4 c h,
$$

where $v=x^{n}$. So

$$
\Delta_{\Delta}^{\prime}=-4 c\left(-a d^{2}-b^{2} c+4 a c h\right) .
$$

Let us note that if $h>\frac{b^{2}}{4 a}+\frac{d^{2}}{4 c}, \Delta_{\Delta}^{\prime}<0$, then $\Delta<0$, and there is no real solution of equation (5).

If $h=\frac{b^{2}}{4 a}+\frac{d^{2}}{4 c}, \Delta_{\Delta}^{\prime}=0$, and $\Delta=-4 a c\left(v+\frac{b}{2 a}\right)^{2}<0$, with $v \neq \frac{-b}{2 a}$, there is no real solution of equation (5).

If $h<\frac{b^{2}}{4 a}+\frac{d^{2}}{4 c}, \Delta_{\Delta}^{\prime}>0$ and we have

$$
\begin{gathered}
v_{1}=\frac{1}{4 a c}\left(\sqrt{4 b^{2} c^{2}-16 a h c^{2}+4 a c d^{2}}-2 b c\right), \\
v_{2}=-\frac{1}{4 a c}\left(\sqrt{4 b^{2} c^{2}-16 a h c^{2}+4 a c d^{2}}+2 b c\right),
\end{gathered}
$$

$\frac{1}{4 a c}\left(\sqrt{4 b^{2} c^{2}-16 a h c^{2}+4 a c d^{2}}-2 b c\right)$ is positive, and if $\frac{d^{2}}{4 c}<h$, then

$$
-\frac{1}{4 a c}\left(\sqrt{4 b^{2} c^{2}-16 a h c^{2}+4 a c d^{2}}+2 b c\right)
$$

is also positive. 
We distinguish the following cases

1) $n$ is odd number. The equation $\Delta=0$ admits two real solutions

$$
\begin{gathered}
x_{1}=\sqrt[n]{-\frac{1}{4 a c}\left(\sqrt{4 b^{2} c^{2}-16 a h c^{2}+4 a c d^{2}}+2 b c\right)}, \\
x_{2}=\sqrt[n]{\frac{1}{4 a c}\left(\sqrt{4 b^{2} c^{2}-16 a h c^{2}+4 a c d^{2}}-2 b c\right)} .
\end{gathered}
$$

For $x \in\left[x_{1}, x_{2}\right]$ the discriminant $\Delta$ is positive and the equation (5) admits two real solutions

$$
z_{1}=\frac{-d-\sqrt{d^{2}-4 c\left(a x^{2 n}+b x^{n}+h\right)}}{2 c}, \quad z_{2}=\frac{-d+\sqrt{d^{2}-4 c\left(a x^{2 n}+b x^{n}+h\right)}}{2 c} .
$$

If $m$ is odd number, they are two real solutions of equation(5) that depend on $y$

$$
\begin{aligned}
& y_{1}=\sqrt[m]{\frac{-d-\sqrt{d^{2}-4 c\left(a x^{2 n}+b x^{n}+h\right)}}{2 c}}, \\
& y_{2}=\sqrt[m]{\frac{-d+\sqrt{d^{2}-4 c\left(a x^{2 n}+b x^{n}+h\right)}}{2 c}},
\end{aligned}
$$

$x \rightarrow y_{1}$ is decreasing function when $\left.x \in\right] x_{1}, \sqrt[n]{\frac{-b}{2 a}}[$ and an increasing function when $x \in] \sqrt[n]{\frac{-b}{2 a}}, x_{2}[$.

$x \rightarrow y_{2}$ is an increasing function when $\left.x \in\right] x_{1}, \sqrt[n]{\frac{-b}{2 a}}[$ and an decreasing function when $x \in] \sqrt[n]{\frac{-b}{2 a}}, x_{2}[$.

Therefore $y_{1}\left(x_{1}\right)=y_{2}\left(x_{1}\right)=\sqrt[m]{\frac{-d}{2 c}}, y_{1}\left(x_{2}\right)=y_{2}\left(x_{2}\right)=\sqrt[m]{\frac{-d}{2 c}}$.

Then $\Gamma$ is composed of an oval in area

$$
D=\left\{(x, y) \in \mathbb{R}^{2}, x_{1}<x<x_{2}, y_{1}\left(\sqrt[n]{\frac{-b}{2 a}}\right)<y<y_{2}\left(\sqrt[n]{\frac{-b}{2 a}}\right)\right\}
$$

If $m$ is even number they are four real solutions of equation(5) that depend on $y: y_{1}, y_{2},-y_{1},-y_{2}$. 
With the same process as before, we conclude that the curve $\Gamma$ is composed of two ovals, one is in the area

$$
D_{1}=\left\{(x, y) \in \mathbb{R}^{2}, x_{1} \leq x \leq x_{2}, y_{1}\left(\sqrt[n]{\frac{-b}{2 a}}\right) \leq y \leq y_{2}\left(\sqrt[n]{\frac{-b}{2 a}}\right)\right\},
$$

and the other is in the area

$$
D_{2}=\left\{(x, y) \in \mathbb{R}^{2}, x_{1} \leq x \leq x_{2},-y_{2}\left(\sqrt[n]{\frac{-b}{2 a}}\right) \leq y \leq-y_{1}\left(\sqrt[n]{\frac{-b}{2 a}}\right)\right\} .
$$

2) $n$ is even number. The equation $\Delta=0$ admits four real solutions $x_{1}, x_{2},-x_{1},-x_{2}$.

For $x \in\left[-x_{2},-x_{1}\right] \cup\left[x_{1}, x_{2}\right]$ the discriminant $\Delta$ is positive and the equation (5) admits two real solutions $z_{1}, z_{2}$.

If $m$ is odd number, they are two real solutions of equation(5) that depend on $y$

$$
\begin{aligned}
& y_{1}=\sqrt[m]{\frac{-d-\sqrt{d^{2}-4 c\left(a x^{2 n}+b x^{n}+h\right)}}{2 c}}, \\
& y_{2}=\sqrt[m]{\frac{-d+\sqrt{d^{2}-4 c\left(a x^{2 n}+b x^{n}+h\right)}}{2 c}} .
\end{aligned}
$$

If $m$ is even number, they are four real solutions of equation(5) that depend on $y: y_{1}, y_{2},-y_{1},-y_{2}$.

As before, we conclude that, when $m$ is odd number, the curve $\Gamma$ is composed of two ovals, one is in the area $D_{1}$, and the other is in the area

$$
D_{3}=\left\{(x, y) \in \mathbb{R}^{2},-x_{2} \leq x \leq-x_{1}, y_{1}\left(-\sqrt[n]{\frac{-b}{2 a}}\right) \leq y \leq y_{2}\left(-\sqrt[n]{\frac{-b}{2 a}}\right)\right\}
$$

when $m$ is even number, the curve $\Gamma$ is composed of four ovals in the areas $D_{1}$, $D_{2}, D_{3}, D_{4}$

$D_{4}=\left\{(x, y) \in \mathbb{R}^{2},-x_{2} \leq x \leq-x_{1},-y_{2}\left(-\sqrt[n]{\frac{-b}{2 a}}\right) \leq y \leq-y_{1}\left(-\sqrt[n]{\frac{-b}{2 a}}\right)\right\}$.

ii) $\Gamma$ is an invariant curve of system (6):

$$
\frac{\partial U}{\partial x} \dot{x}+\frac{\partial U}{\partial y} \dot{y}=\left((x y+P(y))\left(2 n a x^{2 n}+b n x^{n}\right)\right.
$$




$$
\left.+(x y+Q(x))\left(2 m c y^{2 m}+d m y^{m}\right)\right) U
$$

The cofactor is

$$
K(x, y)=(x y+P(y))\left(2 n a x^{2 n}+b n x^{n}\right)+y(x y+Q(x))\left(2 m c y^{2 m}+d m y^{m}\right) .
$$

iii) Let $\int_{0}^{T} \operatorname{div}(\Gamma) d t \neq 0$. Note that

$$
\begin{gathered}
\int_{0}^{T} \operatorname{div}(\Gamma) d t=\int_{0}^{T} K(x(t), y(t)) d t \\
\int_{0}^{T} K(x(t), y(t)) d t=\int_{0}^{T} x(x y+P(y))\left(2 n a x^{2 n-1}+b n x^{n-1}\right) d t \\
+\int_{0}^{T} y(x y+Q(x))\left(2 m c y^{2 m-1}+d m y^{m-1}\right) d t \\
=\oint_{\Gamma} \frac{(x y+P(y))\left(2 n a x^{2 n}+b n x^{n}\right)}{y\left(2 n a x^{2 n}+b n x^{n}\right)} d y-\oint_{\Gamma} \frac{(x y+Q(x))\left(2 m c y^{2 m}+d m y^{m}\right)}{\left(2 m c y^{2 m}+d m y^{m}\right)} d x \\
=\oint_{\Gamma}\left(x+\frac{P(y)}{y}\right) d y-\oint_{\Gamma}\left(y+\frac{Q(x)}{x}\right) d x .
\end{gathered}
$$

Applying the Green formula

$$
\oint_{\Gamma}\left(x+\frac{P(y)}{y}\right) d y-\oint_{\Gamma}\left(y+\frac{Q(x)}{x}\right) d x=\iint_{i n t(\Gamma)} 2 d x d y
$$

where int $\Gamma$ denotes the interior of $\Gamma$. Moreover, as $\iint_{i n t(\Gamma)} d x d y$ is nonzero then $\int_{0}^{T} K(x(t), y(t)) d t$ is also nonzero.

\section{Examples}

Example 1. Let $m=n=1, a=1, c=2, b=-4, d=-5, h=6$, $P(y)=y+1, Q(x)=x-1$,

$$
\begin{gathered}
\dot{x}=x\left(x^{3} y-3 x^{2} y+x^{2}+2 x y^{3}-5 x y^{2}+2 x y-4 x+2 y^{3}-7 y^{2}+6 y+6\right) \\
\dot{y}=y\left(x^{3} y+x^{3}-4 x^{2} y-3 x^{2}+2 x y^{3}-3 x y^{2}+x y+6 x-2 y^{2}+5 y-6\right)
\end{gathered}
$$

The system (12) admits one limit cycle represented by the curve $x^{2}-4 x+2 y^{2}-$ $5 y+6=0$, and it has five singular points, two are saddle points, two are stable focus and one is an instable focus. The limit cycle encloses a stable focus. 


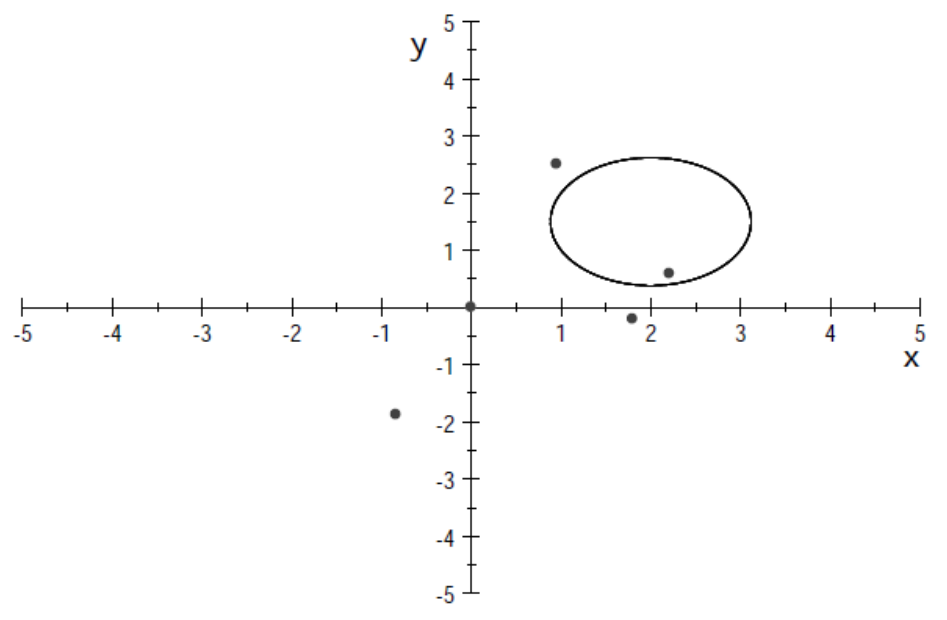

Figure 1: Limit cycle of system (12) with singular points

Example 2. Let $m=1, n=2, a=1, b=-4, c=3, d=-5, h=5$, $P(y)=y^{2}-1, Q(x)=x^{2}+1$

$$
\begin{aligned}
\dot{x}= & x\left(x^{5} y+x^{4} y^{2}-x^{4}-4 x^{3} y-4 x^{2} y^{2}+4 x^{2}+3 x y^{3}-5 x y^{2}+5 x y\right. \\
& \left.+3 y^{4}-5 y^{3}-4 y^{2}+10 y-5\right), \\
\dot{y}= & y\left(x^{6}+x^{5} y+x^{4}-4 x^{3} y+3 x^{2} y^{2}-5 x^{2} y-7 x^{2}\right. \\
& \left.+3 x y^{3}-5 x y^{2}+5 x y+3 y^{2}-5 y+5\right) .
\end{aligned}
$$

The system (13) admits two limit cycles represented by the curve $x^{4}-4 x^{2}+$ $3 y^{2}-5 y+5=0$, and it has eleven singular points, six are saddle points, three are stable focus, two are instable focus, the limit cycle if first quadrant encloses a stable focus, and the other encloses an instable focus.

Example 3. Let $m=n=2, a=3, b=-5, c=2, d=-3, h=3$, $P(y)=y^{2}-3 y+1, Q(x)=x^{2}+x+1$

$$
\begin{aligned}
\dot{x}= & x\left(3 x^{5} y+3 x^{4} y^{2}-9 x^{4} y+3 x^{4}-5 x^{3} y-5 x^{2} y^{2}+15 x^{2} y-5 x^{2}\right. \\
& \left.+2 x y^{5}-4 x y^{3}+3 x y+2 y^{6}-6 y^{5}-10 y^{4}+12 y^{3}+7 y^{2}-9 y+3\right), \\
\dot{y}= & y\left(3 x^{6}+3 x^{5} y+3 x^{5}+13 x^{4}-5 x^{3} y-5 x^{3}+2 x^{2} y^{4}-4 x^{2} y^{2}\right. \\
& -17 x^{2}+2 x y^{5}+2 x y^{4}-4 x y^{3}-4 x y^{2}+3 x y+3 x+4 y^{4}-8 y^{2}+6
\end{aligned}
$$

The system (14) admits four limit cycles, represented by the curve $3 x^{4}-5 x^{2}+$ $2 y^{4}-4 y^{2}+3=0$, and it has ten singular points, four are saddle points, three 


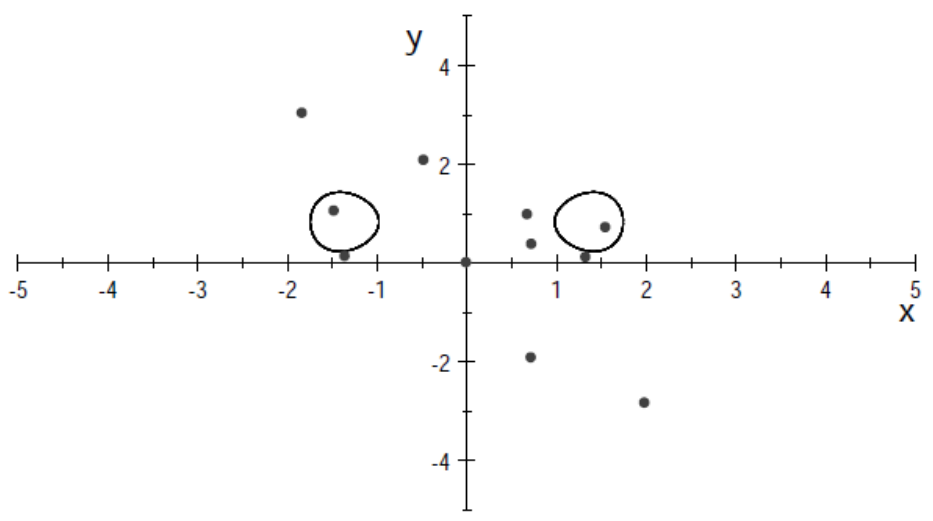

Figure 2: Limit cycle of system (13) with singular points

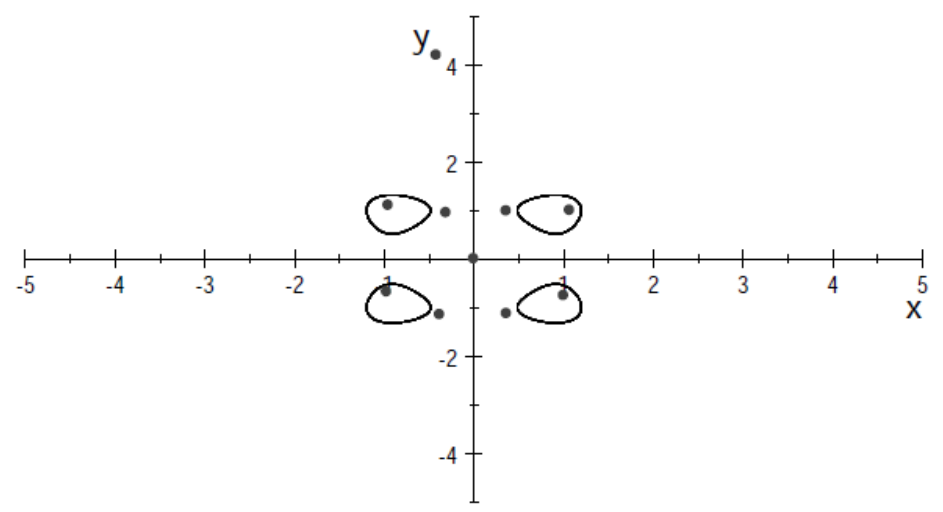

Figure 3: Limit cycle of system (14) with singular points

are stable focus, two are unstable focus and one is unstable node, limit cycles in the first and fourth quadrant around stable focus and limit cycles in the second and third quadrant around unstable focus.

Example 4. Let $m=2, n=, a=2, b=-3, c=1, d=-3, h=3$, $P(y)=\sin (y), Q(x)=\cos (x)$

$$
\begin{aligned}
\dot{x}= & x\left(\sin (y)\left(2 x^{6}-3 x^{3}+y^{4}-3 y^{2}+3\right)\right. \\
& \left.+2 x^{7} y+x y^{5}-3 x^{4} y-3 x y^{3}+3 x y+6 y^{2}-4 y^{4}\right), \\
\dot{y}= & y\left(\cos (x)\left(-3 x^{3}-3 y^{2}+2 x^{6}+y^{4}+3\right)\right. \\
& \left.+2 x^{7} y+x y^{5}-3 x^{4} y-3 x y^{3}+3 x y-9 x^{3}+12 x^{6}\right) .
\end{aligned}
$$




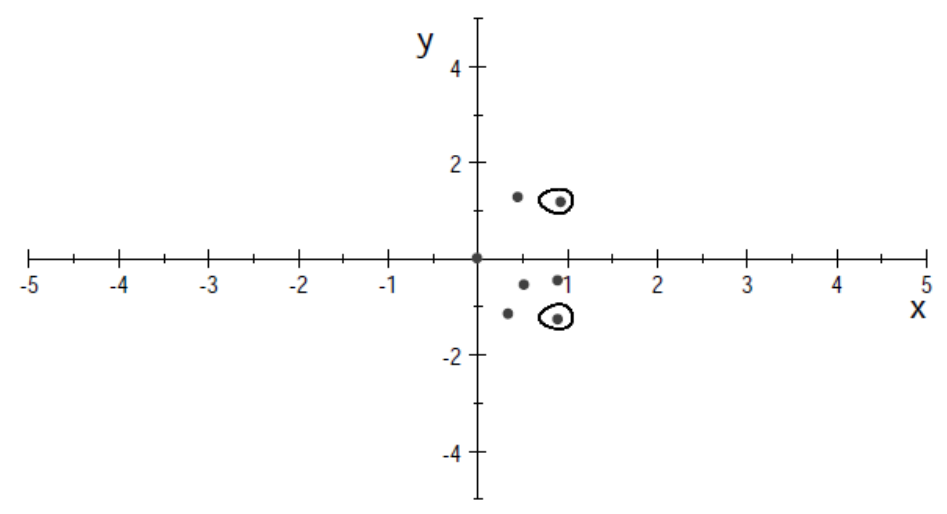

Figure 4: Limit cycle of system (15) with singular points

The system (15) admits two limit cycles represented by the curve $2 x^{6}-3 x^{3}+$ $y^{4}-3 y^{2}+3=0$, the limit cycle in the first quadrant is around an unstable focus and the limit cycle in the fourth quadrant is around a stable focus.

\section{References}

[1] M. Abdelkadder, Relaxation oscillator with exact limit cycles, J. of Math. Anal. and Appl. 218 (1998), 308-312.

[2] M.J. Alvarez , A. Gasull , R. Prohens. Limit cycles for two families of cubic systems. Nonlinear Analysis 75 (2012) pp 6402-6417.

[3] A. Bendjeddou and R. Cheurfa, On the exact limit cycle for some class of planar differential systems, Nonlinear differ. equ. appl. 14 (2007), pp 491-498.

[4] A. Bendjeddou and R. Cheurfa, Cubic and quartic planar differential systems with exact algebraic limit cycles, Elect. J. of Diff. Equ., no15 (2011), pp1-12.

[5] T.R.Blows and N.G.Lloyd, The number of limit cycles of certain polynomial differential equations, proceeding of royal society of edinburgh, section a mathematics/ volume 98 / issue 3-4/ January 1984, pp 215-239.

[6] Shen Boqian and Liu Demeng. Existence of limit cycles for a cubic Kolmogorov system with a hyperbolic solution. Northwest Math.16(1) 2000, pp 91-95. 
[7] J. Chavarriga, H. Giacomini and J. Giné, On a new type bifurcation of limit cycles for a planar cubic systems, Nonlin. Anal.Theo, Meth and Appli 36 (1999),pp 139-149.

[8] K.S. Cheng, Uniqueness of a limit cycle for a predator-prey system, SIAM J. Math. Anal, 12 (1981),541-548.

[9] SI Chengbin, Shen Boqian. The existence of limit cycles for the Kolmogorov cubic system with a quartic curve solution.J.Sys. Sci.\& Math. Scis.28(3) (2008), pp 334-339.

[10] F. Dumortier, J. Llibre and J. Artés, Qualitative Theory of Planar Differential Systems, (Universitex) Berlin, Springer (2006).

[11] H. Giacomini, J. Libre and M. Viano, On the nonexistence, existence, and uniqueness of limit cycles, Nonlinearity 9 (1996), pp 501-516.

[12] J. Giné and M. Grau, A note on: "Relaxation Oscillator with Exact Limit Cycles", J. of Math. Anal. and Appl. 324 no. 1 (2006), pp739-745.

[13] J.Giné, J.Llibre, Integrability and algebraic limit cycles for polynomial differential systems with homogeneous nonlinearities. J Differential Equations, $v 208$ issue 2, 2005, pp 531-545.

[14] Xun Cheng. Huang and Lemin Zhu, Limit cycles in a general Kolmogorov model, Nonlin. Anal. Theo. Meth. and Appl. 60 (2005), 1393-1414.

[15] X.C. HUANG, . Limit cycle in a Kolmogorov-type model, Internat. J. Math. \& Math Sci.VOL. 13 NO. 3 (1990) 555-566

[16] X. Huang, Y. Wang, A. Cheng, Limit cycles in a cubic predator-prey differential system, J. Korean Math. Soc. 43 (2006) 829-843.

[17] Y.Kuang and H.I Freedman, Uniqueness of limit cycles in Gause-type models of Predator-prey systems, Math. Biosci.. 88 issue 1 (1988),pp 67-84.

[18] J. Llibre and Y. Zhao, Algebraic Limit Cycles in Polynomial Systems of Differential Equations, J. Phys. A: Math. Theor. 40 (2007), pp 1420714222.

[19] J.Llibre, R.Ramírez,N.Sadovskaia, On the 16th Hilbert problem for algebraic limit cycles, J. Differential Equations 248 issue 6 (2010) pp 14011409. 
[20] J.Llibre, R.Ramírez,N.Sadovskaia, On the 16th Hilbert problem for limit cycles on nonsingular algebraic curves, J. Differential Equations 250 issue2 (2011) pp 983-999.

[21] N. G. Lloyd and J. M. Pearson, E Saèz and I. Szántó, Limit cycles of a Cubic Kolmogorov System, Appl. Math. Lett. vol 9 No.1,(1996) pp 15-18.

[22] N. G. Lloyd, J. M. Pearson, E. Sáez, and I. Szántó, A cubic Kolmogorov system with six limit cycles, Computers and Mathematics with Applications v 44, issues3-4 (2002), pp 445-455.

[23] R.M. MAY, Limit cycles in predator-prey communities, Science v177no 4052 (1972), pp 900-902.

[24] L. Perko, Differential equations and dynamical systems, Third edition. Texts in Applied Mathematics, 7. Springer-Verlag, New York, 2001.

[25] Y.Q. YE, C.Y Lo. Theory of Limit Cycles. Amer. Math. Soc., Providence, R.I.,1986.

[26] Peng Yue-hui. Limit Cycles in a Class of Kolmogorov Model with Two Positive equilibrium Points. Natural Science journal of Xiangtan University,Vol. 32 No.4 Dec. 2010. 10-15

[27] X.Zhang, The 16th Hilbert problem on algebraic limit cycles, J. Differential Equations 251 (2011) pp17r7-1789. 
